\title{
Image Clustering by Generative Adversarial Optimization and Advanced Clustering Criteria
}

\author{
Eva Tuba, Ivana Strumberger, Nebojsa Bacanin, Timea Bezdan, \\ and Milan Tuba $\left.{ }^{(}\right)$
}

Singidunum University, Danijelova 32, 11000 Belgrade, Serbia

tuba@ieee.org

\begin{abstract}
Clustering is the task that has been used in numerous applications including digital image analysis and processing. Image clustering refers to the problem of segmenting image for different purposes which leads to various clustering criteria. Finding the optimal clusters represented by their centers is a hard optimization problem and it is one of the main research focuses on clustering methods. In this paper we proposed a novel generative adversarial optimization algorithm for finding the optimal cluster centers while using standard and advance clustering criteria. The proposed method was tested on seven benchmark images and results were compared with the artificial bee colony, particle swarm optimization and genetic algorithm. Based on the obtained results, the generative adversarial optimization algorithm founded better cluster centers for image clustering compared to named methods from the literature.
\end{abstract}

Keywords: Image clustering $\cdot$ Swarm intelligence $\cdot$ Generative adversarial optimization

\section{Introduction}

Digital images are a big part of the modern world since they have been part of almost every scientific field as well as an important part of everyday life. There are countless benefits that the usage of digital images has brought. Fast and relatively simple but precise analysis of digital images enabled their usage in some unexpected fields such as agriculture [15] while in some other fields where images have been used even before such as medicine, they brought huge improvements [21]. Besides digital images taken by mobile phones or cameras that capture the visible light which are the most common digital images in everyday life, in science various other modalities are used such as X-ray, ultrasound, magnetic resonance imaging (MRI), etc.

The final goal of digital image processing application can be the detection of plant diseases [9], tumor recognition [3], bleeding detection [20], fire detection [22], and many more. In order to achieve these goals which are rather different, some standard and common image processing methods adjusted for the specific

(C) Springer Nature Switzerland AG 2020

Y. Tan et al. (Eds.): ICSI 2020, LNCS 12145, pp. 465-475, 2020.

https://doi.org/10.1007/978-3-030-53956-6_42 
problem are used. All digital image processing methods and algorithms can be categorized as low, middle or high-level algorithms and usually, methods from more than one category are used in one application. Usually, pre-processing represents the first step in all applications where the digital image is enhanced for further processing. Low-level methods such as contrast adjustment, changing the brightness, binarization of the digital image are frequently used in the pre-processing step and they have a common factor which is that all these methods manipulate with the pixel values without any further understanding of the objects in the image. Methods from the middle-level category represent a higher level of digital image processing where certain characteristics such as shapes, textures, edges and contours are recognized. Again, there is no further knowledge about what is on the image. An example can be detecting faces by finding rounded objects in skin color. High-level digital image processing methods use artificial intelligence for recognizing elements that were detected by methods of low and middle processing levels such as recognizing persons based on the detected faces. Each of the steps, pre-processing, detection and recognition are important for the success of the final application. One of the important tasks in middle-level processing is segmentation. Segmentation is the participation of the digital image into meaningful parts. Due to the similarity of the segmentation and clustering problems, clustering algorithms have been widely used for digital image segmentation. In both cases the goal is to divide data into clusters so the data from one cluster are more similar to each other than to data from other clusters. In the case of segmentation, data can be pixels or regions.

Currently, there are numerous clustering methods proposed and used in various applications such as hierarchical clustering, DBSCAN, many distributions based clustering algorithms, k-means, and others. In this paper we used the modified and optimized k-means algorithm for digital image segmentation. It is wellknown that the quality of clustering by the k-means algorithm is determined by initial cluster centers. Finding the optimal cluster centers is a hard optimization problem and many optimization techniques have been proposed for solving it, including swarm intelligence algorithms $[3,6,19]$. When using swarm intelligence algorithms it is important to define fitness function according to the considered problem. In this paper, a novel swarm intelligence algorithm, generative adversarial optimization is used for finding optimal cluster centers and three different fitness functions have been used, two standards and one proposed especially for digital image segmentation proposed in [13].

The rest of this paper is organized as follows. Short literature review is given in Sect. 2. Section 3 defines the generative adversarial optimization algorithm used for image segmentation along with the used fitness functions. A comparison of the proposed method with other approaches from the literature is presented in Sect. 4. The conclusion of this paper is given in Sect. 5 .

\section{Literature Review}

Image clustering represents common problem in various applications that deal with the standard, medical, satellite or other images. Since it is a very common 
task in image processing applications, numerous methods can be found in the literature.

One of the well-known clustering algorithms is k-means and it was widely used for the image segmentation. The method for 2-level segmentation was proposed in [4]. Before applying the clustering algorithm, images were preprocessed by the partial stretching enhancement method while the initial cluster centers were determined by the subtractive clustering method and the segmentation was enhanced by using median filter. Similar approach was presented in [23] for fish image segmentation.

Even though the k-means algorithm is one of most commonly used clustering algorithms it has one big drawback - it is highly sensitive to the choice of the initial cluster centers. Due to this issue, k-means algorithm is frequently combined by the optimization metaheuristics such as swarm intelligence algorithms.

Combination of the k-means algorithm and the particle swarm optimization and ant colony optimization algorithm was proposed and tested in [11]. In [7], the k-means algorithm was optimized by the firefly algorithm while in [10] instead of the original k-means algorithm, fuzzy k-means method was proposed.

Besides optimizing the k-means algorithm was data clustering, this method was also used for the image segmentation problem. In [2], hybridization of the particle swarm optimization and the k-means algorithm was proposed in [2] and it was used for the image segmentation. The obtained segmentation was further enhanced by the spatial gray-level information used for the correction of misclassified pixels.

The gravitation search algorithm was used to automatically determine the number of segments, i.e. clusters and to optimize the k-means algorithm for the image segmentation problem.

The k-means algorithm was also used for medical image applications in $[5,14,17]$. In [14] adaptive k-means algorithm was used for breast cancer detection while in [5] fuzzy k-means algorithm combined by the genetic algorithm and particle swarm optimization was applied to noisy MRI images. The method used in [5] was based on the results presented in [1] where different soft computing methods were combined by the fuzzy k-means algorithm and applied to MRI image segmentation. Another method for the medical image segmentation was proposed in [16] where genetic algorithm used for optimizing the k-means algorithm and the proposed method was applied to brain tumor detection in MRI images.

\section{Generative Adversarial Optimization for Image Clustering}

The generative adversarial optimization (GAO) algorithm represents one of the recent swarm intelligence optimization algorithm proposed by Tan and Shi in 2019 [18]. Currently, there are only two papers that apply the GAO algorithm for support vector machine optimization [21] and image compression [8]. In the paper where the GAO algorithm was presented it was tested on standard benchmark 
functions for bound constrained optimization problems. Based on the comparison with the other optimization methods from the literature, it was concluded that the GAO is a competitive metaheuristic for solving hard optimization problems. While the majority of the optimization metaheuristics use random sampling or guiding vectors for generating new solutions, the GAO algorithm has a drastically different approach by using the generative adversarial network as an inspiration. In the GAO algorithm, a generator is used for searching new solutions and the discriminator tries to predict if the new solution is better than the previous one. Feedback from the discriminator is used as the guiding vector that provides better solutions. The GAO algorithm is presented in Fig. 1.

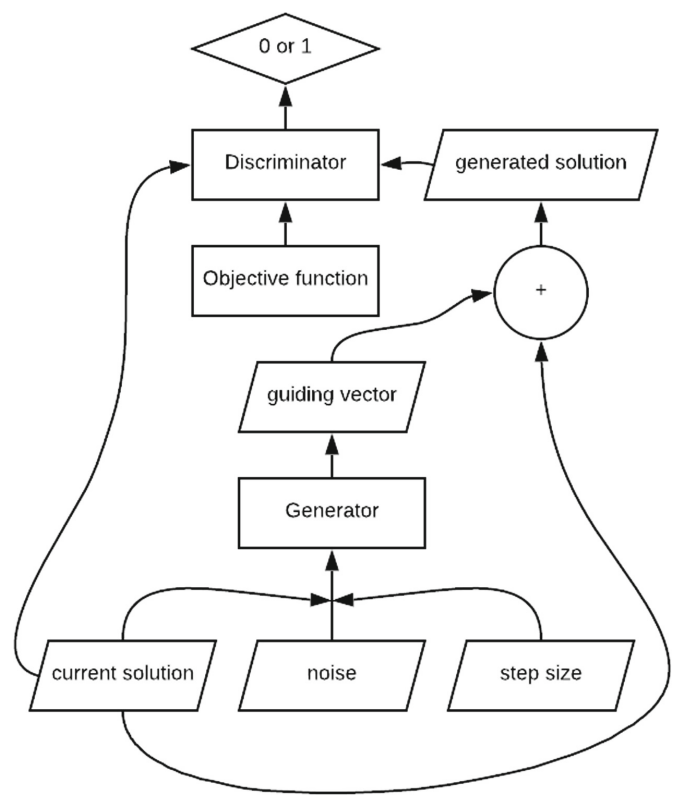

Fig. 1. The GAO algorithm

Inputs for the generator $G$ are the current solution denoted as $x_{c}$, a noise vector $z$ and the step size $l$. Output produced by the generator represents a guiding vector $g$, i.e. $g=G\left(x_{c}, z, l\right)$. The whole population is updated based on the output of the generator $G$ presented in Fig. 2(a). As it can be seen from the Fig. 2(a), guided vector $g$ is obtained after the concatenation of the current solution and the noise used as an input for fully-connected layer denoted by FC whose output is combined with the step size $l$ :

$$
g=G\left(x_{c}, z, l\right)=F C\left(\left[x_{c}^{T}, z^{T}\right]^{T}\right) \cdot l .
$$

In the GAO algorithm, new solutions are generated by adding the guiding vector $g$ to the current solution $x_{c}$. 
After generating new solutions, the discriminator $D$ is used to make a prediction is the new solution better than the current solution. Labels used for the training are set by the following function:

$$
y^{i}= \begin{cases}1, & f\left(x_{g}\right)<f\left(x_{c}\right) \\ 0, & \text { else }\end{cases}
$$

where $x_{g}$ is generated solution and $f$ is objective function. The discriminator's scheme is shown in Fig. 2(b), The discriminator $D$ contains two fully-connected layers denoted by $F C 1$ and $F C 2$. Inputs for the $F C 1$ are the current $x_{c}$ and generated solutions $x_{g}$. The output of the $F C 1$ is subtracted from $x_{g}$ and that is the input for the $F C 2$. The output of $D$ represents a prediction if the new solution improves the value of the objective function.

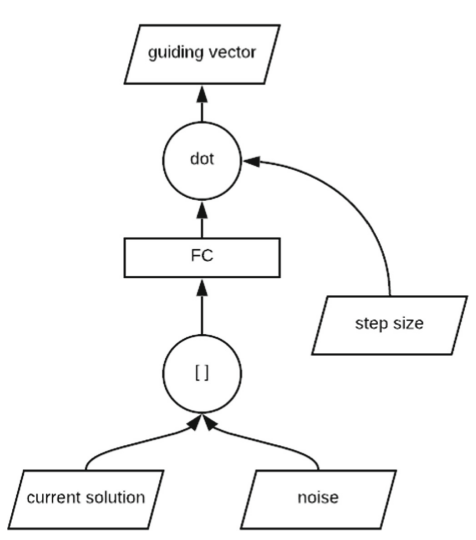

(a) GAO generator

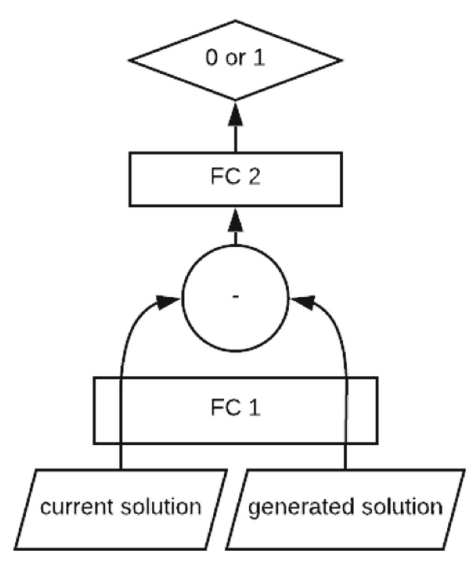

(b) GAO discriminator

Fig. 2. The GAO generator and descriptor

In this paper, we used the GAO algorithm for finding the optimal cluster centers for image clustering task. The dimension of the problem is equal to the number of clusters. Pixels are assigned to the cluster which center is closest to it by theirs pixel intensity values. Solutions are ranked based on three different objective functions.

\subsection{Objective Functions}

In clustering algorithms there are three main criteria that are used for determining the quality of the clusters, comprising error measure, inter-cluster distance and intra-cluster separation. The first fitness function that will be used 
as objective function for the generative adversarial optimization algorithm combines these three criteria in weighted sum [12]:

$$
f_{1}\left(x_{i}, Z\right)=w_{1} d_{\max }\left(Z, x_{i}\right)+w_{2}\left(z_{\max }-d_{\min }\left(Z, x_{i}\right)\right)+w_{3} J_{e},
$$

where $Z$ denotes the image i.e. set of pixel values, $x_{i}$ is the $i^{\text {th }}$ solution which is $K$ cluster centers, $z_{\max }$ represents the maximal intensity value of the pixels in the image which is for the s-bit image equal to $2^{s}-1$. Parameters $w_{1}, w_{2}$ and $w_{3}$ are redetermined weights that controls the influence of each criterion. Value of $d_{\max }$ represents the maximal average distance between pixels and their corresponding centers:

$$
d_{\text {max }}\left(Z, x_{i}\right)=\max _{k}\left(\sum_{\forall z_{p} \in C_{i, k}} \frac{d\left(z_{p}, m_{i, k}\right)}{n_{i, k}}\right),
$$

where $n_{i, k}$ is total number of elements in the cluster $C_{i, k}$ and $d\left(z_{p}, m_{i, k}\right)$ represents Euclidean distance between pixel $z_{p}$ and cluster center $m_{i, k}$. The value of $d_{\min }$ in the Eq. 3 represents the minimal average Euclidean distance between any two clusters:

$$
d_{\text {min }}\left(Z, x_{i}\right)=\min d\left(m_{i, j}, m_{i, l}\right), \quad j \neq l, j, l=1,2,3, \ldots, K .
$$

The third part of the first objective function is quantization error $J_{e}$ defined as:

$$
J_{e}=\frac{\sum_{k=1}^{K} \sum_{\forall z_{p} \in C_{k}} d\left(z_{p}, m_{k}\right) / n_{k}}{K} .
$$

This quantization error is used to determine the overall quality of the clustering.

The second objective function that is considered in this paper is more flexible compared to the first one since there are no parameters (except the number of clusters) that need to be set. It is defined as:

$$
f_{2}\left(x_{i}, Z\right)=\frac{d_{\max }\left(Z, x_{i}\right)+J_{e, i}}{d_{\min }\left(Z, x_{i}\right)} .
$$

The third objective function was proposed in [13]. This objective function was proposed with intention to include benefits while eliminating drawbacks of the previous two fitness functions. The third objective function uses mean square error combined by the quantization error. It was designed to maximize $d_{\max }$ while minimizing $d_{m i n}$. Definition of the third objective function is as follows:

$$
f_{3}\left(x_{i}, Z\right)=J_{e} \frac{d_{\max }\left(Z, x_{i}\right)}{d_{\min }\left(Z, x_{i}\right)}\left(d_{\max }\left(Z, x_{i}\right)+z_{\max }-d_{\min }\left(Z, x_{i}\right)+M S E\right),
$$

where $M S E$ is the mean square error of cluster centers and actual pixel values. 


\section{Experimental Results}

The method proposed for digital image clustering by generative adversarial optimization algorithm was tested on Intel (R) Core ${ }^{\mathrm{TM}}$ i7-3770K CPU at $4 \mathrm{GHz}, 8 \mathrm{~GB}$ RAM, Windows 10 Professional OS computer. The proposed GAO clustering method was implemented in Python 3.7.

The proposed GAO clustering method is compared to other state-of-theart methods presented in [13]. In [13] the proposed objective function defined by Eq. 8 was used with the artificial bee colony (ABC) algorithm while it was compared by particle swarm optimization (PSO) and genetic algorithm (GA). Images used in [13] have been used in our test too. Test images are standard benchmark images: airplane, house, Lena, Morro Bay and MRI along with two images from the Berkeley segmentation dataset named 42049 and 48025. Test images are shown in Fig. 3. The number of clusters $K$ for all images was set to 5 . Parameters for the ABC, PSO and GA are listed in [13]. The weights for the first objective function were set as follow: $w_{1}=0.4, w_{2}=0.2, w_{3}=0.4$. Parameters of the GAO algorithm were set based on the recommendations in [18]: a total number of solutions kept for the next iteration was $n=5$ while in each iteration $\beta=30$ new solutions are generated and the selection process control parameter was $\alpha=2$. Fine tuning of the parameters can be the part of future work since it is known that adequate choice for the parameters is the crucial for the optimal results of any optimization algorithm. For each image, the algorithm was started 30 times. Comparisons of the results obtained for different fitness functions are presented in Table 1, Table 2 and Table 3.

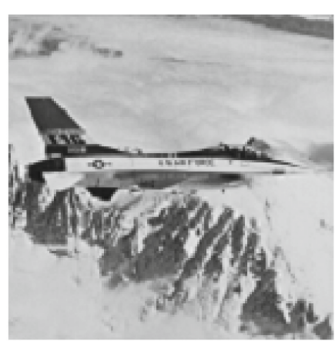

(a)

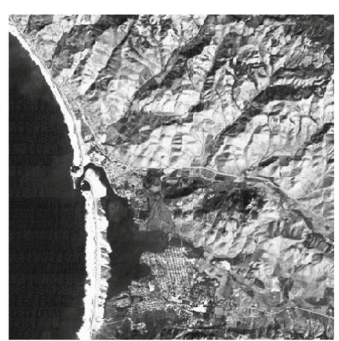

(b)

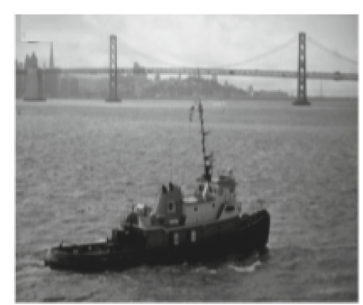

(c)

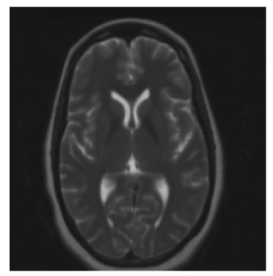

(d)

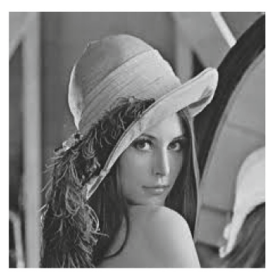

(e)

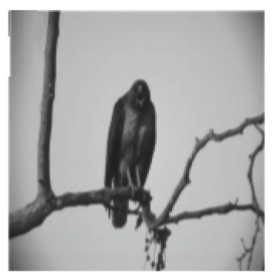

(f)

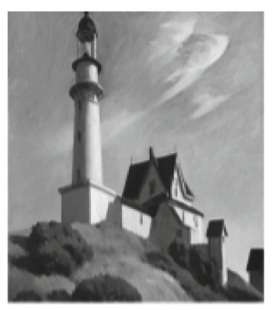

(g)

Fig. 3. Test images 
Table 1. Comparison of the results with the first objective function of the proposed method and method proposed in [13]

\begin{tabular}{|c|c|c|c|c|}
\hline & GA & PSO & $\mathrm{ABC}$ & GAO \\
\hline Air & 47.328 & $46.275(0.273)$ & $46.159(0.0$ & $46.124(0.283)$ \\
\hline House & $51.732(0.993)$ & $51.116(0.841)$ & $50.795(0.1$ & $50.623(0.314)$ \\
\hline Lena & $50.666(0.616$ & $50.087(C$ & $50.065(0.0$ & $50.049(0.113)$ \\
\hline Morro & 50.7 & 50.1 & $50.097(0.1$ & $50.083(0.167)$ \\
\hline MRI & $40.929(0.466)$ & $40.448(0.096)$ & $40.490(0.073)$ & $40.442(0.089)$ \\
\hline 42049 & $52.089(1.065$ & $50.768(0.170)$ & $50.789(0.0$ & $50.7^{\prime}$ \\
\hline 48025 & $1.675(0.562)$ & $51.074(0.277)$ & $51.064(0.104)$ & $51.072(0.197)$ \\
\hline
\end{tabular}

Based on the results presented in Table 1, we can see that the proposed GAO method outperformed all three algorithms used for comparison for standard benchmark images, while for the images from the Berkeley dataset PSO and ABC methods obtained slightly better results. It should be noticed that for all test images (also for all objective functions) ABC algorithm has a smaller standard deviation compared to the proposed GAO algorithm. This should be further investigated and possibly outcast this drawback. One of the possible solution is fine tuning the GAO parameters and adjusting them to the considered problem rather than using the same parameters as in [18] were the GAO was applied to benchmark functions.

In the case when the second fitness function was sed (Eq. 7), our proposed GAO and ABC have comparable results. For the images airplane, house and Morro bay, the proposed GAO method found better solutions while for the other test images, the ABC method achieved better results but just for 0.001 .

Table 2. Comparison of the results with the second objective function of the proposed method and method proposed in [13]

\begin{tabular}{|c|c|c|c|c|}
\hline & GA & PSO & $\mathrm{ABC}$ & GAO \\
\hline Airplane & $0.570(0.059$ & $0.500(0.013)$ & $0.498(0.005)$ & $0.496(0.007)$ \\
\hline House & $0.545(0.053)$ & $0.505(0.047)$ & $0.490(0.008)$ & $0.483(0.007)$ \\
\hline Lena & $0.544(0.044)$ & $0.503(0.011)$ & $0.503(0.006)$ & $0.504(0.003)$ \\
\hline Morro Bay & 0.48 & 0.45 & $0.454(0$ & $0.450(0.00$ \\
\hline MRI & 0.490 & $0.448(0.005)$ & $0.446(0.003)$ & 0.447( \\
\hline 42049 & $0.603(0$. & 0.488 & $0.487(0.0$ & $0.488(0.007)$ \\
\hline 48025 & $0.531(0.029)$ & $0.506(0.016)$ & $0.505(0.007)$ & $0.505(0.009)$ \\
\hline
\end{tabular}

At the end, we used the objective function proposed in [13] defined by Eq. 8 . It can be noticed that the values of the fitness function are much higher compared 
to the cases when the first and the second objective function were used. When the third objective function is used, PSO outperformed ABC for airplane, Lena and 42049 images while ABC outperformed the proposed GAO algorithm only for Morro Bay image.

Table 3. Comparison of the results with the third objective function of the proposed method and method proposed in [13]

\begin{tabular}{|c|c|c|c|c|}
\hline & GA & $\mathrm{PSO}$ & $\mathrm{ABC}$ & GAO \\
\hline Airplane & 924.596 (133.109) & $777.767(8.676)$ & $790.618(8.789)$ & $768.248(9.103)$ \\
\hline House & $1192.324(212.851)$ & $1085.34(137.140)$ & $1070.080(11.840)$ & $1062.982(33.345)$ \\
\hline Lena & $1025.065(100.002)$ & $899.365(11.464)$ & 919.307 (13.987) & $881.634(15.239)$ \\
\hline Morro Bay & $998.267(130.435)$ & 898.866 (64.648) & $886.052(9.825)$ & $887.213(14.920)$ \\
\hline MRI & $595.781(61.695)$ & $523.751(16.786)$ & $523.145(7.282)$ & 522.928 (12.837) \\
\hline 42049 & $714.839(45.818)$ & $667.083(9.110)$ & $673.370(7.785)$ & $664.396(12.441)$ \\
\hline 48025 & $1332.130(202.390)$ & $1202.15(24.536)$ & $1192.57(16.602)$ & $1186.751(20.498)$ \\
\hline
\end{tabular}

\section{Conclusion}

Digital images found their purpose in various scientific fields which resulted by numerous research works and applications in this domain. One of the common tasks in digital image processing applications is segmentation which can be done by clustering algorithms. In this paper we proposed usage of novel generative adversarial optimization algorithm for finding optimal cluster centers. The proposed method was tested with three different fitness functions and compared to the genetic algorithm, particle swarm optimization and artificial bee colony algorithm. The best results were obtained while using standard clustering objective function and improved clustering fitness. Based on the results, it can be concluded that the proposed GAO algorithm is more suitable for image clustering tasks compared to other state-of-the-art methods.

\section{References}

1. Agrawal, S., Panda, R., Dora, L.: A study on fuzzy clustering for magnetic resonance brain image segmentation using soft computing approaches. Appl. Soft Comput. 24, 522-533 (2014)

2. Benaichouche, A.N., Oulhadj, H., Siarry, P.: Improved spatial fuzzy c-means clustering for image segmentation using pso initialization, mahalanobis distance and post-segmentation correction. Digit. Sig. Process. 23(5), 1390-1400 (2013)

3. Capor Hrosik, R., Tuba, E., Dolicanin, E., Jovanovic, R., Tuba, M.: Brain image segmentation based on firefly algorithm combined with k-means clustering. Stud. Inform. Control 28, 167-176 (2019)

4. Dhanachandra, N., Manglem, K., Chanu, Y.J.: Image segmentation using k-means clustering algorithm and subtractive clustering algorithm. Procedia Comput. Sci. 54, 764-771 (2015) 
5. Forouzanfar, M., Forghani, N., Teshnehlab, M.: Parameter optimization of improved fuzzy c-means clustering algorithm for brain MR image segmentation. Eng. Appl. Artif. Intell. 23(2), 160-168 (2010)

6. Hancer, E., Ozturk, C., Karaboga, D.: Artificial bee colony based image clustering method. In: Congress on Evolutionary Computation (CEC), pp. 1-5. IEEE (2012)

7. Hassanzadeh, T., Meybodi, M.R.: A new hybrid approach for data clustering using firefly algorithm and k-means. In: The 16th CSI International Symposium on Artificial Intelligence and Signal Processing (AISP 2012), pp. 007-011. IEEE (2012)

8. Huang, C., Liu, H., Chen, T., Shen, Q., Ma, Z.: Extreme image coding via multiscale autoencoders with generative adversarial optimization. In: 2019 IEEE Visual Communications and Image Processing (VCIP), pp. 1-4. IEEE (2019)

9. Lukic, M., Tuba, E., Tuba, M.: Leaf recognition algorithm using support vector machine with $\mathrm{Hu}$ moments and local binary patterns. In: 2017 IEEE 15th International Symposium on Applied Machine Intelligence and Informatics (SAMI), pp. 000485-000490. IEEE (2017)

10. Nayak, J., Nanda, M., Nayak, K., Naik, B., Behera, H.S.: An improved firefly fuzzy C-means (FAFCM) algorithm for clustering real world data sets. In: Kumar Kundu, M., Mohapatra, D.P., Konar, A., Chakraborty, A. (eds.) Advanced Computing, Networking and Informatics- Volume 1. SIST, vol. 27, pp. 339-348. Springer, Cham (2014). https://doi.org/10.1007/978-3-319-07353-8_40

11. Niknam, T., Amiri, B.: An efficient hybrid approach based on PSO, ACO and k-means for cluster analysis. Appl. Soft Comput. 10(1), 183-197 (2010)

12. Omran, M.G., Engelbrecht, A.P., Salman, A.: Particle swarm optimization for pattern recognition and image processing. In: Abraham, A., Grosan, C., Ramos, V. (eds.) Swarm Intelligence in Data Mining, vol. 34, pp. 125-151. Springer, Heidelberg (2006). https://doi.org/10.1007/978-3-540-34956-3_6

13. Ozturk, C., Hancer, E., Karaboga, D.: Improved clustering criterion for image clustering with artificial bee colony algorithm. Pattern Anal. Appl. 18(3), 587-599 (2014). https://doi.org/10.1007/s10044-014-0365-y

14. Patel, B.C., Sinha, G.: An adaptive k-means clustering algorithm for breast image segmentation. Int. J. Comput. Appl. 10(4), 35-38 (2010)

15. Shen, Q., Kirschbaum, M.U., Hedley, M.J., Arbestain, M.C.: Testing an alternative method for estimating the length of fungal hyphae using photomicrography and image processing. PloS One 11(6), e0157017 (2016)

16. Sinha, K., Sinha, G.: Efficient segmentation methods for tumor detection in MRI images. In: Conference on Electrical, Electronics and Computer Science, pp. 1-6. IEEE (2014)

17. Sulaiman, S.N., Isa, N.A.M.: Adaptive fuzzy-K-means clustering algorithm for image segmentation. IEEE Trans. Consumer Electron. 56(4), 2661-2668 (2010)

18. Tan, Y., Shi, B.: Generative adversarial optimization. In: Tan, Y., Shi, Y., Niu, B. (eds.) ICSI 2019. LNCS, vol. 11655, pp. 3-17. Springer, Cham (2019). https://doi. org/10.1007/978-3-030-26369-0_1

19. Tuba, E., Dolicanin-Djekic, D., Jovanovic, R., Simian, D., Tuba, M.: Combined elephant herding optimization algorithm with K-means for data clustering. In: Satapathy, S.C., Joshi, A. (eds.) Information and Communication Technology for Intelligent Systems. SIST, vol. 107, pp. 665-673. Springer, Singapore (2019). https:// doi.org/10.1007/978-981-13-1747-7_65

20. Tuba, E., Tuba, M., Jovanovic, R.: An algorithm for automated segmentation for bleeding detection in endoscopic images. In: International Joint Conference on Neural Networks (IJCNN), pp. 4579-4586. IEEE (2017) 
21. Tuba, M., Tuba, E.: Generative adversarial optimization (GOA) for acute lymphocytic leukemia detection. Stud. Inform. Control 28(3), 245-254 (2019)

22. Tuba, V., Capor-Hrosik, R., Tuba, E.: Forest fires detection in digital images based on color features. Int. J. Educ. Learn. Syst. 2 (2017)

23. Yao, H., Duan, Q., Li, D., Wang, J.: An improved k-means clustering algorithm for fish image segmentation. Math. Comput. Model. 58(3-4), 790-798 (2013) 